\section{S-469 AN INTEGRATED DATABASE OF OCCUPATIONAL INFORMATION FROM O*NET-SOC AND THE CANADIAN CAREER HANDBOOK}

'Vadym Babiuk, Anil Adisesh, Christopher JO Baker. 'University of New Brunswick, Canada

10.1136/OEM-2021-EPI.443

Introduction Information about occupations and job attributes is available in siloed databases. The lack of integrated data precludes ad-hoc querying and research investigating occupational determinants of health e.g. COVID-19 or stress. Core to integration of occupation data is taxonomic representation of job categories. In North America the official occupational taxonomies are the Canadian National Occupational Classification (NOC) and the United States Standard Occupational Classification (SOC).

Objectives This study aimed to integrate job attribute data from the Canadian Career Handbook $(\mathrm{CH})$ and $\mathrm{O}^{*} \mathrm{NET}$ database to facilitate cross-classification query capabilities and to prototype the creation of metrics for comparing occupations based on job attributes.

Methods

The integrated database was completed hierarchical structures of both occupational taxonomies were represented; job attributes were selected from the $\mathrm{CH}$ and $\mathrm{O} * \mathrm{NET}-\mathrm{SOC}$; the database was populated with occupational descriptions; occupational codes from the $\mathrm{CH}$ and $\mathrm{O} * \mathrm{NET}-\mathrm{SOC}$ were linked using the Brookfield Institute NOC to O*NET-SOC crosswalk.

Results The database consists of 1679 rows with unique occupations and 181 columns with occupational attributes. Rows contain a unique combination of hierarchical structures from the $\mathrm{CH}$ and $\mathrm{O}^{*}$ NET-SOC. Rows also contain detailed occupational descriptions from $\mathrm{CH}$ and $\mathrm{O}$ *NET-SOC. We queried the integrated data checking $\mathrm{O} * \mathrm{NET}-\mathrm{SOC}$ to $\mathrm{CH}$ equivalence and cross-taxonomy selection of job attributes, e.g. Retrieve all or selected attributes for an occupation by $\mathrm{CH}$ code or equivalent code in $\mathrm{O}^{*} \mathrm{NET}-\mathrm{SOC}$. We ran queries for targeted scenarios to retrieve occupations: i) where work is done in physical proximity to others, ii) where incumbents are exposed to disease or infections, iii) at risk of back pain due to physical work factors, iv) where incumbents experience high workrelated stressors.

Conclusion We report a database combining selected information from the $\mathrm{CH}$ and $\mathrm{O} * \mathrm{NET}-\mathrm{SOC}$ that facilitates complex occupational health queries. Further we investigated workrelated stressors on low back pain risk by occupation.

\section{S-487 YOUNG PEOPLE'S DEPRESSIVE SYMPTOM TRAJECTORIES AND THEIR EDUCATION AND EMPLOYMENT. COMPARING CANADA AND THE UNITED STATES}

${ }^{1}$ Anita Minh, Ute Bültmann, Sijmen Reijneveld, Sander van Zon, Chris McLeod. 'University of British Columbia, Canada

\subsection{6/OEM-2021-EPI.444}

Objective This study examines how trajectories of depressive symptoms from the age of 16-25 are related to early adult education and employment outcomes in Canada and the United States.

Methods Data came from the Canadian National Longitudinal Survey of Children and Youth $(n=2348)$ and the American
National Longitudinal Survey of Youth 1979 Child/Young Adult Survey $(n=3961)$. Depressive symptom trajectories from the age of 16-25 were identified separately for each country using growth-mixture modeling, and linked to respondents' education and employment status (working with a post-secondary degree; working with no degree; working with a high school degree; in school; and, not in employment, education, or training i.e., NEET), and part/full-time employment (less than 30 hours/week, 30-40 hours/week, more than 40 hours/week). We assessed the association of depressive symptom trajectories with these outcomes using multivariable multinomial logistic regressions, calculating the adjusted predicted probability of each outcome using marginal standardization.

Results In both countries four similar depressive symptom trajectories were identified: low-stable, increasing, decreasing, and first increasing then decreasing symptoms (i.e., mid-peak). In both countries, increasing, decreasing, and mid-peak trajectories were associated with higher odds of working with low educational credentials, and/or NEET relative to low-stable trajectories. In Canada, however, all trajectories had a higher predicted probability of either being in school or working with a post-secondary degree than the other outcomes; in the USA, all trajectory groups were most likely to be working with a high school degree. In the USA but not in Canada, increasing and decreasing trajectories were associated with higher odds of part-time work than full-time work.

Conclusions Higher levels of depressive symptoms during the transition to adulthood are associated with working with no or low credentials, NEET, and working part-time in young adulthood. Country-level differences may modify the influence of depressive symptoms.

\section{S-490 EXPOSOME METHODS IN OCCUPATIONAL EPIDEMIOLOGY: USE OF TEXT MINING FOR DEVELOPING JOB EXPOSURE MATRICES}

${ }^{1}$ Martie van Tongeren, Calvin Ge, Eelco Kuijpers, Sophia Ananiadou, Hakan Tinnerberg, Annika Schoene, Ioannis Basinas, Susan Peters, Anjoeka Pronk. 'The University of Manchester, United Kingdom

\subsection{6/OEM-2021-EPI.445}

Within the EXPOSOME PROJECT FOR HEALTH AND OCCUPATIONAL RESEARCH (EPHOR) project we aim to develop a protocol to enable efficient update of job exposure matrices so that they can include the latest available information of highest quality possible. The protocol will include methods for searching and collecting new data from literature (assisted by text mining WP4), exposure databases (e.g. ECHA REACH database, reports) and (Bayesian) decision criteria to determine if and how to revise exposure estimates in the JEM. As part of this work we have started to develop a framework of semi- and fully-automated approaches for identification of relevant literature and extraction of occupational exposure measurements, which in turn may be used in creating and updating JEMs. Currently both content-level and document-level approaches are being explored. The contentlevel approach utilizes text-mining and machine learning to interpret, analyse, and return relevant information from a text corpus (e.g. manuscripts in the PubMed Central (PMC) archive). In addition to retrieval of user-specified information (e.g. 'literature with occupational benzene measurements in 
PMC form 2018-2020'), the software will also have the potential to identify new patterns and relationships within the corpus (e.g. 'the most sampled industry/occupation in literature with benzene measurements in PMC from 20182020'). The document-level method uses automated keyword searches with optional filters to highlight documents with potential relevant information. Once highlighted by the search algorithm, the documents may be screen manually or with other automatic software to extract relevant information and data. We are currently focusing on exposure to diesel engine exhaust but plan to expand to other substances. The protocol will form part of the EPHOR toolbox being developed as part of the project.

\section{S-492 A RESEARCH PROGRAM TO PROVIDE A COMPARATIVE CONTEXTUALIZED ANALYSIS OF OCCUPATIONAL COVID-19 AMONG HEALTH WORKERS: PRELIMINARY INSIGHTS FROM A SOUTH AFRICAN-CANADIAN COLLABORATION}

${ }^{1}$ Jerry M Spiegel, Annalee Yassi, Muzimkhulu Zungu, Sipho Senabe, Spo Kgalamono, David Jones, Nisha Naicker, Arnold Okpani, Jennifer Grant, Rodney Ehrlich, Andrés DelgadoRon, Hillary Mukuduh. ${ }^{1}$ University of British Columbia, Canada

10.1136/OEM-2021-EPI.446

A research program to provide a comparative contextualized analysis of occupational COVID-19 among health workers: Preliminary insights from a South African-Canadian Collaboration.

Objective The COVID-19 pandemic has demonstrated that healthcare workers (HCWs) in many settings are at high risk of occupational exposure to infectious diseases, especially where attention to occupational protection was lacking. In July 2020 our World Health Organization (WHO) collaborating centres in Canada and South Africa launched a joint Rapid Response Research program in partnership with local government health service delivery agencies in both countries to better understand how local contexts affect policies and practice; scrutinize their respective scientific and contextual rationales as well as outcome; grasp why and how these change over time; and understand organizational factors that enhance implementing resilient policies.

Methods The collaboration includes cohort studies, in the Vancouver Coastal Health $(\mathrm{VCH})$ region in Canada, and Gauteng province in South Africa respectively, to assess risk factors for SARS-CoV-2 infection among HCWs as well as evaluate the effectiveness of SARS-CoV-2 infection prevention and control measures. It also includes a cross-sectional study in Gauteng to explore mental health of HCWs during the pandemic and identify areas for intervention; a quasi-experimental study of the role of information systems in strengthening occupational health services for healthcare workers; and global policy analyses including an analysis of a global survey of HCWs from 161 countries.

Results The global survey revealed considerable variations in the degree to which prevention and control measures were deemed adequate; the South African baseline audit of 42 hospitals also revealed considerable variations in implementing occupational health protection. We demonstrated the utility of information systems to assess risk by occupation and setting in $\mathrm{VCH}$; preliminary results of the $\mathrm{VCH}$ case-control study demonstrated the feasibility of this design; and, importantly, we identified challenges in leveraging operational research to inform policy, practice and world-knowledge in both $\mathrm{VCH}$ and South Africa.

Conclusion Our research activities showed the impact of vaccine roll-out and new variants on rates of COVID-19 among HCWs within different healthcare settings and occupational groups and how policies to protect HCWs have evolved (e.g., masking policies and vaccine protocols for HCWs). We conclude that lessons regarding procedural barriers to data acquisition and sharing must be addressed with an ethical framework in mind.

\section{S-493 ARTIFICIAL INTELLIGENCE AND OCCUPATIONAL HEALTH \\ ${ }^{1} \mathrm{R}$ Vermeulen. ${ }^{1}$ Utrecht University, Netherlands}

10.1136/OEM-2021-EPI.447

The multitude and complexity of data in health sciences has given rise to the increasing use of artificial intelligence (AI). AI technologies of importance include machine (ML) and deep (DL) learning, natural language processing (NLP) and rule-based expert systems (RBES). These AI technologies have also found their way into occupational health in the analyses of structured and unstructured data varying from application in job-codings (e.g. NLP). exposure assessment (e.g. NLP and RBES), data analyses (e.g. ML, DL and Neural networks), and risk assessment (RBES). Examples of AI technologies in Occupational Health will be presented including efforts on job-codings, job-exposure-matrix construction from the EPHOR project, high-dimensional (ML) data analyses within UK-Biobank and the Synergy project, and the application of RBES in risk assessment of benzene. Ethical issues in the application of AI in occupational health will also be discussed.

\section{S-494 MECHANISTIC EVIDENCE FROM POPULATION STUDIES OF WELDING FUME EXPOSURE AND CANCER RISK}

${ }^{1} \mathrm{D}$ Christiani. ${ }^{1}$ Harvard School of Public Health, United States

\subsection{6/OEM-2021-EPI.448}

Objectives to describe evidence from human studies of welding fume associated responses predisposing to cancer.

Methods The process of evaluating potential carcinogenicity of occupational exposures entails comprehensive evaluation of in vitro and in vivo studies of cells, animals and human populations. With advancements in our ability to assess exposurerelated health affects in humans, we can use molecular epidemiologic methods to study pre-clinical disruptions in homeostasis that can lead to cancer. Welding fumes were evaluated recently in IARC Monograph \#118 (2017).

Results With respect to the key characteristics of human carcinogens, adequate data from in vitro and in vivo studies were available to evaluate if welding fumes: induce chronic inflammation; are immunosuppressive; are genotoxic; induce oxidative stress; alter cell proliferation, cell death, and nutrient supply; and modulate receptor-mediated effects. There is 